BMJ Open

Sport \&

Exercise

Medicine

\section{Age-related changes in the effects of strength training on lower leg muscles in healthy individuals measured using MRI}

To cite: Psatha M, Wu Z, Gammie F, et al. Age-related changes in the effects of strength training on lower leg muscles in healthy individuals measured using MRI. BMJ Open Sport Exerc Med 2017;3:e000249. doi:10.1136/bmjsem-2017000249

Accepted 20 June 2017

CrossMark

For numbered affiliations see end of article.

Correspondence to Dr Richard M Aspden, Institute of Medical Sciences, School of Medicine, Medical Sciences and Nutrition, University of Aberdee, Foresterhill, Aberdeen AB25 2ZD, UK; r.aspden@abdn.ac. uk

\section{ABSTRACT}

Background We previously measured the rate of regaining muscle strength during rehabilitation of lower leg muscles in patients following lower leg casting. Our primary aim in this study was to measure the rate of gain of strength in healthy individuals undergoing a similar training regime. Our secondary aim was to test the ability of MRI to provide a biomarker for muscle function.

Methods Men and women were recruited in three age groups: 20-30, 50-65 and over 70 years. Their response to resistance training of the right lower leg twice a week for 8 weeks was monitored using a dynamometer and MRI of tibialis anterior, soleus and gastrocnemius muscles at 2 weekly intervals to measure muscle size (anatomical cross-sectional area $(A C S A))$ and quality ( $T_{2}$ relaxation). Forty-four volunteers completed the study.

Results Baseline strength declined with age. Training had no effect in middle-aged females or in elderly men in dorsiflexion. Other groups significantly increased both plantarflexion and dorsiflexion strength at rates up to $5.5 \mathrm{~N} \mathrm{~m}$ week ${ }^{-1}$ in young females in plantarflexion and $1.25 \mathrm{~N} \mathrm{~m}$ week ${ }^{-1}$ in young males in dorsiflexion. No changes were observed in ACSA or $T_{2}$ in any age group in any muscle.

Conclusion Exercise training improves muscle strength in males at all ages except the elderly in dorsiflexion. Responses in females were less clear with variation across age and muscle groups. These results were not reflected in simple MRI measures that do not therefore, provide a good biomarker for muscle atrophy or the efficacy of rehabilitation.

\section{INTRODUCTION}

Sarcopenia is defined as a degenerative loss of muscle mass and function with age. ${ }^{1}$ Muscle disuse, as in cast immobilisation following an injury, can also induce loss of muscle independently of ageing. The rate of loss is considerably more rapid than in agerelated sarcopenia. ${ }^{2}$ In a previous study, we found that immobilisation resulted in a loss

\section{What are the new findings}

- The rate of gain in strength of healthy individuals following a strength training regime is less than that experienced during a similar rehabilitation programme.

- Normalised responses (standardised response means) were smaller in dorsiflexion than plantarflexion.

Middle-aged females showed no response to training.

\section{Impact on clinical practice}

Gains in strength through training are less certain in older individuals, especially in females, and different training regimes should be explored to be tailored to each individual depending on their age and sex.

- Assessment of muscle training cannot be done sensitively using MRI.

of more than $25 \%$ of muscle volume, ${ }^{3}$ but once the cast was removed, muscle volume was rapidly restored so that by the end most measures were comparable with those from the contralateral leg. ${ }^{4}$ We wish to know how the restoration of atrophied muscle compares with the ability of healthy muscles to gain strength when subjected to similar course of strength training.

MRI can delineate soft tissues, such as muscle, and measure markers of chemical and structural changes non-invasively and so has great potential for the development of imaging biomarkers of physiological processes or pathology. One indicator of muscle quality is the $T_{2}$ value since it relates to various aspects of the muscle such as fibre type, ${ }^{5}$ water content and organisation, 
and fat content. $^{6}$ Sarcopenia, especially in those muscles containing mainly type 2 fibres, such as the gastrocnemius, has been associated with lengthening $T_{2}$ relaxation times. ${ }^{5}$ Inactivity has been associated with decreases in strength and increases in fat in young $^{7}$ and older people, ${ }^{8}$ with exercise training reducing the quantity of intramuscular fat. ${ }^{8}$ Exercise also affects $T_{2}$ with reports of an increase after strenuous exercise returning to baseline values with a halflife of around $8 \mathrm{~min} .{ }^{9}$ This response depends on the dominant fibre type, and muscles containing mostly type 2 fibres are said be most responsive. ${ }^{10}$ Atrophy of the quadriceps through unweighting was found to result in greater than normal $T_{2}$ changes with exercise. $^{11}$

We wish to know how does restoration after disuse compare with the effects of training on normal individuals from their normal baseline? In parallel with the study of patients, ${ }^{4}$ we investigated the ability of healthy individuals to strengthen their lower leg muscles. We also explored the utility of MRI to quantify age-related differences in the response of muscle to training. To do this, we recruited healthy men and women in three different age groups and monitored the response of their lower leg muscles to resistance training. We used a dynamometer to measure their strength and MRI to measure muscle size and quality.

\section{METHODS}

\section{Participants}

We recruited 48 healthy volunteers, in three equalsized groups, by advertising locally for volunteers in three age groups: 20-30, 50-65, and over 70 years of age. The oldest group came from a local 'evergreens' fitness class; the remainder were largely staff in our institutions. Participants had to be self-reported as 'healthy' and were excluded if they reported taking medication within the previous 2 years that might affect muscle function, had diabetes, uncontrolled thyroid disease, previous or current musculoskeletal morbidity in the legs, or the usual contraindications to MRI including pregnancy, claustrophobia, body mass index (BMI) over $35 \mathrm{~kg} / \mathrm{m}^{2}$, implanted metalwork or a cardiac pacemaker. Each volunteer was given a verbal description of the study and prescreened. Those still interested and thought suitable were offered a study information sheet. Those willing to participate were then invited to attend for full screening, give their written consent and have their first study visit at which their height, weight and any medication use were also recorded. All procedures in this study were performed in accordance with the 1964 Helsinki Declaration and its later amendments. The study was approved by the North of Scotland Research Ethics Committee (Reference 08/S0801/101), and all participants gave their written, informed consent.

\section{Study design}

Participants undertook an 8-week period of strength training, attending twice each week for a programme of isokinetic exercise, and underwent strength assessment every 2 weeks. They were invited for MRI examinations on entry to the study and at the end of weeks $2,4,6$ and 8 , coinciding with the strength assessment.

\section{MRI protocols}

Both legs were imaged simultaneously in a Philips Achieva 3.0 T whole body MRI scanner using a Philips 16 channel SENSE XL Torso coil. Patients were positioned supine, feet first, with the knees extended but relaxed. The torso coil was positioned under and over both legs so the scan could span the length of the lower leg. A custom-made Perspex frame and Velcro straps were used to keep the feet and legs close together. The tibial tuberosity and the inferior tip of lateral malleolus were used as primary reference landmarks to identify positions of the scans. External markers were placed at points corresponding to $50 \%$ and $70 \%$ of this distance proximal to the distal marker using cod liver oil softgel capsules $(1 \mathrm{~cm}$ diameter, $2.4 \mathrm{~cm}$ length). The $50 \%$ marker was used to position the subject in the middle of the scanner and the $70 \%$ position, which corresponds to the bulkiest part of the calf muscles, was used for reproducible slice positioning during image acquisition and for subsequent analysis. A high-resolution 3D $T_{1}$-weighted gradientecho image was used to provide an anatomical reference for subsequent scans. Full details of the imaging protocols have been presented elsewhere. ${ }^{3}$ Quality assurance for MRI was performed monthly using two phantoms to ensure accuracy and precision.

\section{Image analysis}

Images were analysed using tools developed using Matlab (The Mathworks, Natick, Massachusetts, USA) and Java to measure cross-sectional areas and $T_{2}$ relaxation times. ${ }^{3}$ Regions of interest were defined in each muscle by segmenting the image around the muscle boundaries, and all measurements were made in the same way on both legs.

To measure muscle volume and anatomical crosssectional area $(A C S A) T_{1}$-weighted spin-echo images (repetition time: $T R=568 \mathrm{~ms}$, echo time: $T E=20 \mathrm{~ms}$, acquisition matrix: $508 \times 362)$ were acquired in two blocks of 20 slices (slice thickness: $2.5 \mathrm{~mm}$ and spacing: $10 \mathrm{~mm}$ ) to span the length of the lower leg. The ACSA were measured at the $70 \%$ position for the main dorsiflexor of the foot, tibialis anterior $(T A)$ and the plantarflexors gastrocnemius medialis and lateralis together $(G M L)$, and soleus $(S O L)$. Total muscle volume was calculated as the product of the sum of these $A C S A$ s and the distance between the most distal part of the gastrocnemius and the most proximal part 
of the tibial plateau and expressed as a fractional change from baseline. This was found previously to reflect accurately the fractional change in the true muscle volume. ${ }^{3}$

$T_{2}$ relaxation times were measured in each of $T A$, $G M L$ and $S O L$ from -1 /slope of the regression line of the natural $\log$ of the signal versus TE. Eight spin echoes were recorded $(T R=3000 \mathrm{~ms}, \quad T E$ $8 \times 10 \mathrm{~ms}$ ), The acquisition matrix was $200 \times 94$, slice thickness was $5.0 \mathrm{~mm}$ and spacing was $5.0 \mathrm{~mm}$. Following $T_{2}$ imaging in rested muscle, an individual exercised strenuously before climbing back onto the bed for a second $T_{2}$ scan. The exercise protocol consisted of repeated two-legged heel-raises performed at a rate of 80 raises per minute. The duration of exercise was determined during the first visit when volunteers exercised until they reached rating 15 on the BORG scale of perceived exertion ${ }^{12}$ (corresponding to heavy exercise; at this level the participant finds the exercise hard). This protocol was designed to exercise maximally each individual while taking account of initial differences in fitness level between individuals. On average, the exercise lasted for about 2 min with the subject returning to the scanner within 3-4 min of the exercise ending.

\section{Strength training and assessment}

Participants attended an 8-week schedule of supervised strength training of the right lower leg, with two training sessions a week following a protocol of isokinetic exercise training using a KIN-COM dynamometer. Training and assessment were done lying supine with the leg secured and an additional strap around the waist. The right foot was secured to the footplate with the lateral malleolus aligned with the axis of rotation, and the footplate was set at a resting position of $90^{\circ}$ with a constant lever arm of $210 \mathrm{~mm}$. Participants were asked to perform maximal plantarflexion between $100^{\circ}$ and $120^{\circ}$ of ankle flexion at an angular speed of $45^{\circ} \mathrm{s}^{-1}$ followed by maximal dorsiflexion to return the ankle to the starting position at a speed of $60^{\circ} \mathrm{s}^{-1}$. The exercise training protocol comprised 6 sets of 10 maximal movement repetitions with $1 \mathrm{~min}$ recovery between each. Strength was assessed by measuring maximum torque at fixed ankle positions for maximum isometric voluntary contraction (MVC): $90^{\circ}$ for plantarflexion and $105^{\circ}$ for dorsiflexion. ${ }^{13}$ Participants were instructed to exert their maximum effort for $5 \mathrm{~s}$, first in plantarflexion followed immediately by dorsiflexion. This was performed three times at 1 min intervals.

\section{Statistical analysis}

Data were tested for normality, and normally distributed data are shown as mean (SD); otherwise the median $(25 \%, 75 \%)$ is given. Differences between left and right limbs and before-and-after comparisons were explored using paired-sample tests. Differences between before and after training were analysed using two-way analysis of variance (ANOVA) with age and sex followed by pair-wise comparison using a Holm-Sidak test. Increases in strength were compared using the standardised response mean (SRM) as a measure of effect size (the difference between week 8 and baseline measures divided by the SD of the difference) and the slope of the regression line of strength versus time (torque increment). The SRM scales the magnitude of the change by the SD of the differences and measures of different magnitudes to be compared. Mean differences lying within the $95 \%$ CIs of the distribution of differences were deemed not significant. Statistical and plotting software used was Sigmaplot for Windows V.12 and V.13 (Systat Software).

\section{RESULTS}

\section{Recruitment}

Forty-eight volunteers were recruited; three withdrew early and a further individual was excluded because they were unable to attend regularly for scans. Details of the remaining 44 are given in table 1 .

\section{Muscle strength}

The average MVC isometric torque in plantarflexion and dorsiflexion over the 8-week training was

Table 1 Demographic details of volunteers who successfully completed the study. Data are shown as mean (SD)

\begin{tabular}{|c|c|c|c|c|c|c|c|c|c|}
\hline \multirow[b]{2}{*}{ Age group } & \multicolumn{3}{|c|}{ Male } & \multicolumn{3}{|c|}{ Female } & \multicolumn{3}{|c|}{ Total } \\
\hline & $20-30$ & $50-65$ & $70+$ & $20-30$ & $50-65$ & $70+$ & $20-30$ & $50-65$ & $70+$ \\
\hline $\mathrm{N}$ & 7 & 7 & 8 & 9 & 7 & 6 & 16 & 14 & 14 \\
\hline Age (years) & $25(3)$ & $58(6)$ & $74(4)$ & $25(3)$ & $53(4)$ & $72(2)$ & $25(3)$ & $55(5)$ & $73(3)$ \\
\hline Height (cm) & $182(10)$ & $173(9)$ & $172(4)$ & $168(5)$ & $162(5)$ & $161(6)$ & $174(10)$ & $167(9)$ & $167(7)$ \\
\hline Weight (kg) & $79(10)$ & $76(5)$ & $79(7)$ & $62(9)$ & $62(16)$ & $70(8)$ & $70(13)$ & $69(14)$ & $75(9)$ \\
\hline BMI (kg/m) & $24(2)$ & $26(3)$ & 27 (3) & $22(4)$ & $24(6)$ & $27(2)$ & $23(3)$ & $25(5)$ & 27 (3) \\
\hline Postmenopausal & - & - & - & $0 / 9$ & $5 / 7$ & $6 / 6$ & - & - & - \\
\hline
\end{tabular}

BMI, body mass index. 
measured at 2 weekly assessments (figure 1). Training induced an increase in strength from baseline to week 8 in plantarflexion that did not depend on age $(p=0.54)$ or sex $(p=0.40)$. In dorsiflexion, there was a significant interaction between age and $\operatorname{sex}(\mathrm{p}<0.001)$. Within each age group, the difference between the sexes was not significant in either dorsiflexion- or plantarflexion apart from the young males and females in dorsiflexion $(p<0.001)$ (table 2$)$. For each sex considered separately, differences in baseline strength with age were not statistically significant.

The effect of training was explored in more detail in each group by calculating the slope of the linear regression equation of MVC torque on study week and from the SRM (table 2). For plantarflexion, training had the greatest effect on female subjects in the young age group, with a torque increment of $5.5 \mathrm{~N} \mathrm{~m}$ week $^{-1}$. Young males, however, showed large variation in their response (figure 2) and as a group their SRM was half that of the young females, and the torque increment was only $3.3 \mathrm{~N} \mathrm{~m}$ week $^{-1}$ (table 2). In dorsiflexion, the roles were reversed with young males showing the greatest effect of training and an SRM three times greater than that of the females. The average torque increment and the SRM in males in plantarflexion was reasonably consistent with age. In females, the torque increment decreased with age, but the SRM dipped at middle age, due to larger variability. In dorsiflexion, young and middle-aged females and elderly males did not gain in strength (table 2), but the other groups gained in strength with torque increments that changed little with ageing.

\section{MRI measures}

Training significantly increased total muscle volume by $4.6 \%(95 \%$ CI $\pm 4.0 \%)$ in the young males. This compared with changes in the untrained leg of $1.7 \%$ $(95 \%$ CI $\pm 4.6 \%)$. Changes in all other groups were not significant; all less than $2 \%$ and within the corresponding $95 \%$ CIs. The resting values for $T_{2}$ increased significantly $(\mathrm{p}<0.001)$ with age (table 3$)$ but showed no indication of changing over the period of the study in response to training; all differences were within the $95 \%$ CI of the distribution of the data. The exerciseinduced increase in $T_{2}$ was highly significant for $G M L$ and SOL $(\mathrm{p}<0.001$, ANOVA) and significant even for $T A(\mathrm{p}=0.036)$ after allowing for age although this increase was smaller (table 4 ).

\section{DISCUSSION}

For comparison with a rehabilitation programme for atrophied lower leg muscles, healthy volunteers of different ages were invited to participate in a similar training regime. Although some groups showed significant increases in measures of muscle strength, these were smaller than those in the rehabilitation programme, were not consistent with age and sex and were poorly reflected in MRI measures of muscle size
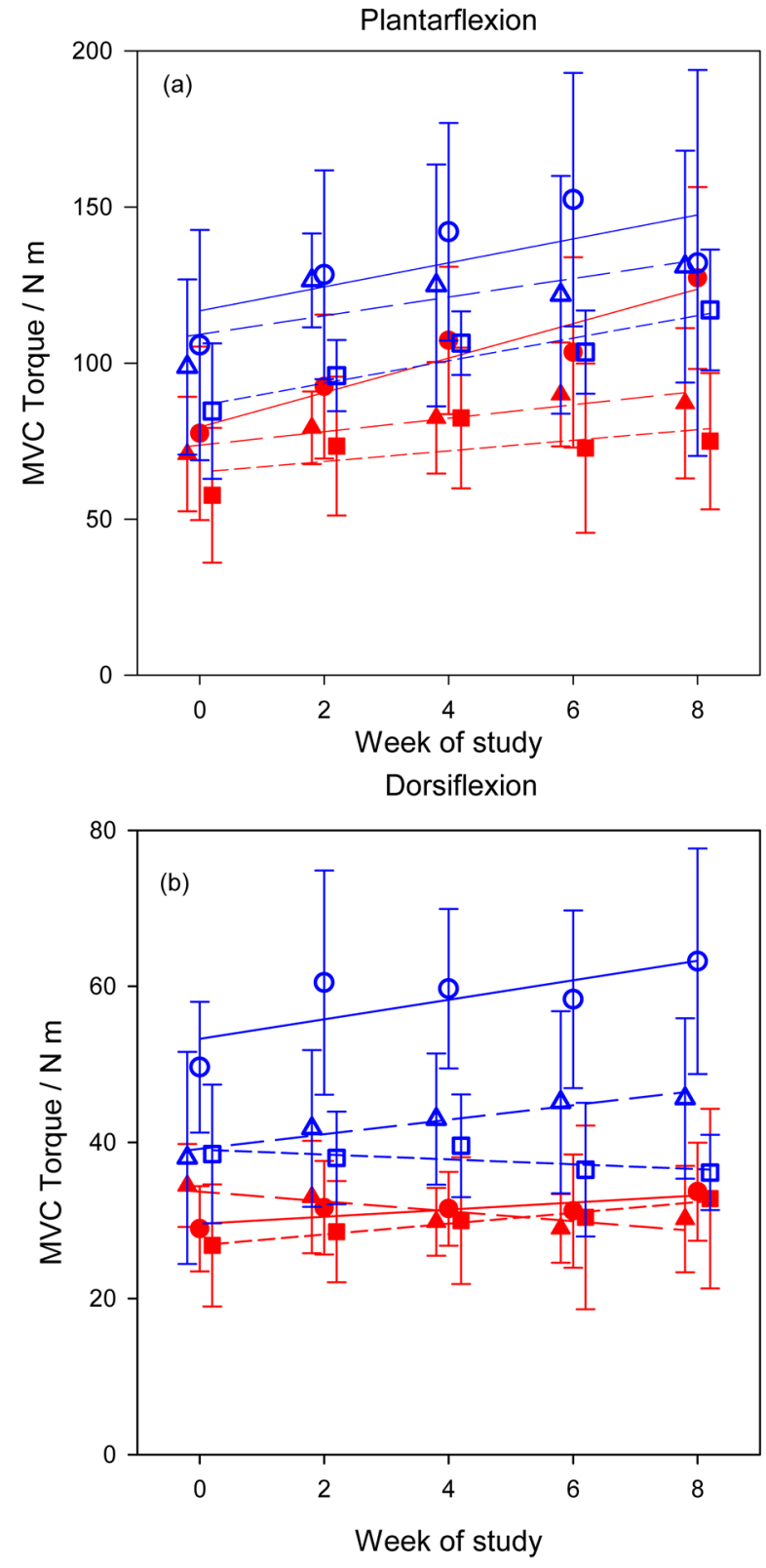

\begin{tabular}{|c|c|c|c|}
\hline - & Young female & 0 & Young male \\
\hline$\Delta$ & Middle-aged female & $\Delta$ & Middle-aged male \\
\hline a & Old female & $\frac{\square}{---}$ & Old male \\
\hline
\end{tabular}

Figure 1 Maximum isometric torque measured at 2 weekly intervals (offset at each time point for clarity of display) (A) at $90^{\circ}$ in plantarflexion and (B) $105^{\circ}$ in dorsiflexion. Linear regression lines were fitted to each age/sex group. MVC, maximum isometric voluntary contraction.

and composition. Changes in muscle ACSA were nearly all not significant, young males being the exception, and no changes were found in pennation angle or water contents (data not shown).

In plantarflexion, all the groups showed an increase in strength over the 8-week period. In males, the average increase changed little with age, although because the variability decreased, the SRM increased. 
Table 2 Mean (SD) MVC torque values recorded on entry and following 8 weeks of isokinetic exercise 2 weekly. Because the magnitudes of the torques are so different in plantarflexion and dorsiflexion, the effect size is shown in terms of SD of the differences using the SRM. The significance of the change following training was determined for each age and sex using a paired-sample $t$-test with a null hypothesis of no change. The final column shows the mean (SE of the mean) of the torque increment derived from the gradients of the regression lines in figure 1

\begin{tabular}{|c|c|c|c|c|c|}
\hline & \multicolumn{4}{|c|}{ Plantarflexion } & \multirow[b]{2}{*}{$\begin{array}{l}\text { Gradient } \\
\text { N m week }\end{array}$} \\
\hline & $\begin{array}{l}\text { Entry } \\
\mathrm{N} \mathrm{m}\end{array}$ & $\begin{array}{l}8 \text { weeks } \\
\mathrm{N} \mathrm{m}\end{array}$ & SRM & $\mathbf{p}$ & \\
\hline Young males & $112(37)$ & $139(54)$ & 0.93 & 0.050 & $3.34(2.29)$ \\
\hline Middle-aged females & $71(18)$ & $87(24)$ & 0.61 & 0.16 & $2.16(0.54)$ \\
\hline Elderly females & $55(23)$ & $75(22)$ & 2.27 & 0.007 & $1.71(1.32)$ \\
\hline \multirow[t]{2}{*}{ Elderly males } & $85(22)$ & 117 (19) & 1.56 & 0.006 & $3.62(0.72)$ \\
\hline & \multicolumn{5}{|l|}{ Dorsiflexion } \\
\hline Young females & $28.9(5.5)$ & $32.1(6.8)$ & 0.49 & 0.18 & $0.45(0.16)$ \\
\hline Elderly females & $27.0(8.7)$ & $32.8(11.5)$ & 1.69 & 0.020 & $0.69(0.08)$ \\
\hline Elderly males & $38.5(8.9)$ & $36.2(4.8)$ & -0.25 & 0.53 & $-0.31(0.19)$ \\
\hline
\end{tabular}

MVC, maximum isometric voluntary contraction.

The young and the elderly females showed a greater average responsiveness than the males with an SRM greater than that of the males. However, the females lost this advantage in the middle-aged group. In dorsiflexion, the results are more varied as the middle-aged females and the elderly males did not gain strength over the study period and the young females had a smaller SRM than the other groups.

Small group sizes and large variations meant that differences in baseline strength were not significant between age groups. Individuals were selected randomly and likely reflect the heterogeneity of the general population, but this meant there was a large overlap in strength between groups; for example, one 81-year-old male in the elderly group was apparently stronger than a 56-year-old male in the middle-aged group. This may be an example where biological age is not wholly reflected in calendar age.

Exercise training was done at relatively slow angular velocities. Pilot experiments showed these slow velocities were better suited for exercising lower leg muscles predominated by slow-contracting type 1 fibres compared with $180^{\circ} \mathrm{s}^{-1}$, which was used in strength training of quadriceps containing equal proportions of type 1 and type 2 fibres. ${ }^{14} 15$

MRI has the potential to be an imaging biomarker as it is sensitive to tissue composition as well as providing excellent soft tissue contrast. The increase in baseline
$T_{2}$ with age could reflect changes in fibre composition as we found no measurable change in MRI measures of fat content (data not shown). Interestingly, the responsiveness of $T_{2}$ to strenuous exercise also increased slightly with age. The increase in measured $T_{2}$ is most likely due to an increase in oedema secondary to an intense rise in blood flow induced by exercise, although it could also be affected by a slower decay of $T_{2}$ values back to baseline while the volunteer clambers back into the scanner. There is some evidence that people who are sedentary with fattier muscles have longer recovery times. ${ }^{16}$ The minimal $T_{2}$ change seen immediately following exercise in $T A$ may suggest we were not as successful at exercising $T A$ compared with $S O L$ and $G M L$.

Studies of muscle function in volunteers are very demanding, often requiring prolonged periods of immobilisation or consistent attendance for training and assessment. In our previous study of rehabilitation using strength training, ${ }^{4}$ we reported a torque increment equivalent to $7.70 \mathrm{~N} \mathrm{~m}$ week $^{-1}$ in males and $5.2 \mathrm{~N}$ $\mathrm{m}$ week $^{-1}$ in females in plantarflexion and in dorsiflexion, $2.5 \mathrm{~N} \mathrm{~m}$ week $^{-1}$ in males and $1.6 \mathrm{~N} \mathrm{~m}$ week $^{-1}$ in females. The males in that study were predominantly young (average age 31 years), whereas the females were closer to middle aged (average age 53 years). In both corresponding age/sex groups, the torque increment was greater in the rehabilitation study, indicating 

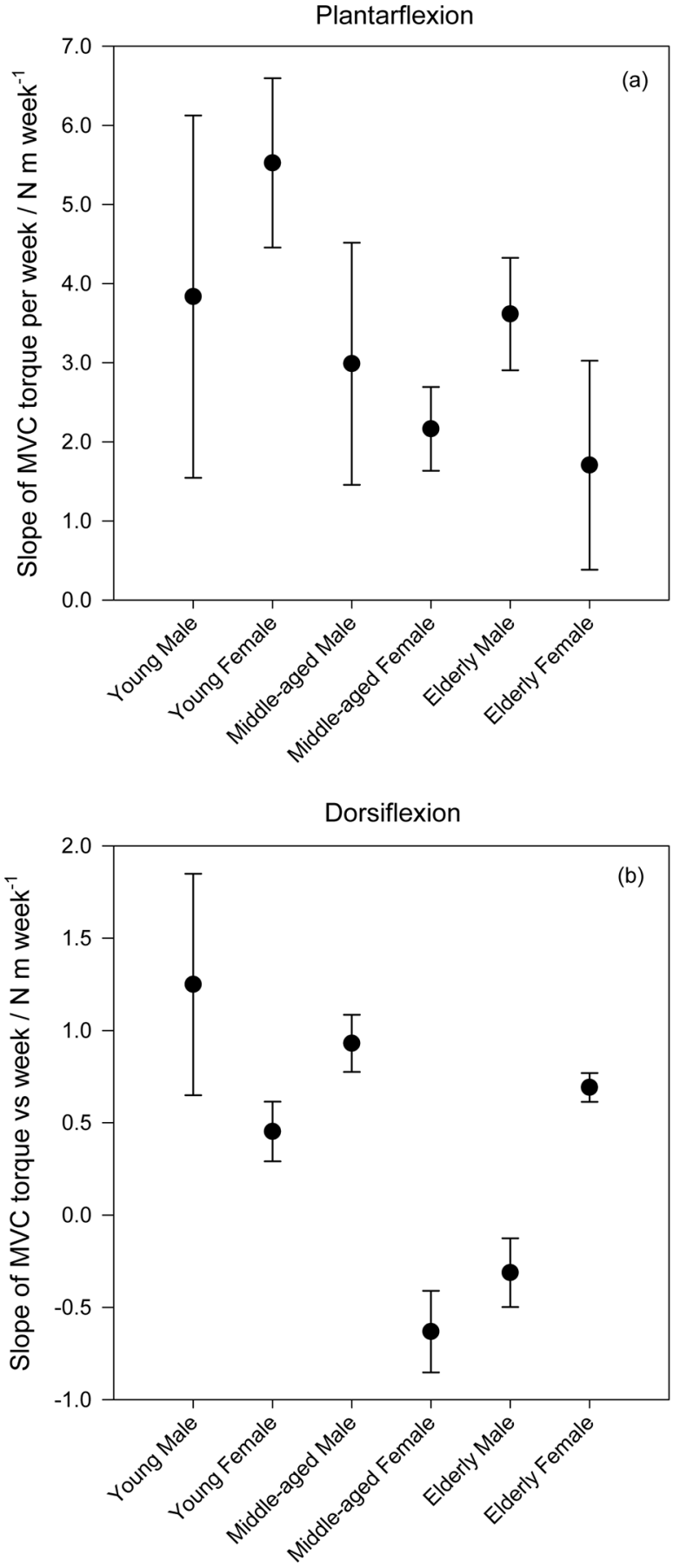

Figure 2 The mean torque increment per week was calculated from the regression lines fitted to the 2 weekly data for each age and sex shown in figure 1. The mean and $\mathrm{SE}$ of the mean of the slopes of the lines are plotted for each group in (A) plantarflexion and (B) dorsiflexion. MVC, maximum isometric voluntary contraction.

that restoring muscle strength to baseline after immobilisation-induced loss may be easier than increasing it from baseline.

Although loss of muscle mass is associated with a reduction in strength, the rate of decline in strength in
Table 3 Mean (SD) $T_{2}$ values for the three muscle groups in each of the age groups. Averages are taken over both legs as there was no apparent effect of training. Overall in each muscle $p<0.001$ and only in TA was there no significant change between middle age and elderly

\begin{tabular}{llll}
\hline & Young & Middle aged & Elderly \\
\hline TA/ms & $25.6(2.2)$ & $27.4(2.6)$ & $27.2(2.8)$ \\
SOL/ms & $33.2(1.3)$ & $35.3(1.5)$ & $37.6(2.9)$ \\
GML/ms & $32.1(1.3)$ & $34.1(1.7)$ & $36.0(2.9)$ \\
\hline
\end{tabular}

$S O L$, soleus; $T A$, tibialis anterior.

sarcopenia has been shown to be much greater than the rate of mass loss. ${ }^{17}$ In lower leg immobilisation, a reduction in strength of $27 \%$ has been reported when muscle mass loss is only about $5 \%{ }^{18}$ We found previously that when compared with values after a period of rehabilitation, a $25 \%$ loss of muscle volume corresponded to a $60 \%$ loss of torque in dorsiflexion and a $50 \%$ loss in plantarflexion. ${ }^{4}$ Conversely, 12 months of resistance training in elderly men (70-82 years) was found to increase plantarflexor MVC torque by $20 \%$, whereas muscle volume assessed by MRI increased by only $12 \%{ }^{19}$ A meta-analysis of studies of resistance training in older people found that none of the measures of training volume correlated well with measures of muscle morphology, and up to a year of training with two to three sessions per week was required to produce measurable improvements in strength and morphology. ${ }^{20}$ Another study found significant increases in quadriceps CSA following 9 weeks of strength training when comparing the trained with the untrained limb. Men displayed greater increases than women but age had little effect. ${ }^{21}$

A similar lack of correspondence is reported in this study in which although muscle strength can be seen to increase, even over 8 weeks, there is no measurable change in muscle volume. It is not clear if the apparent mismatch is due to changes in specific muscle tension (force per cross sectional area) or impaired ability to activate the skeletal muscle. It does, however, indicate that the quality of the muscle is affected and that changes in muscle mass or ACSA alone do not provide a good

Table 4 Differences (mean (SD)) in $T_{2}$ values measured before and immediately after exercise in each muscle in each of the age groups. All differences were significant: $G M L$ and $S O L(p<0.001), T A(p=0.036)$ allowing for differences in age

\begin{tabular}{llll}
\hline & Young & Middle aged & Elderly \\
\hline TA/ms & $0.1(1.2)$ & $0.2(1.8)$ & $0.4(1.4)$ \\
SOL/ms & $1.2(1.3)$ & $1.7(1.3)$ & $1.8(1.8)$ \\
GML/ms & $3.2(1.3)$ & $4.6(1.5)$ & $4.3(1.6)$ \\
\hline
\end{tabular}

SOL, soleus; $T A$, tibialis anterior. 
biomarker to monitor the progression of muscle atrophy or the efficacy of rehabilitation. Larger numbers may clarify these results, but recruiting and retaining volunteers for such an intensive study was not easy.

\section{CONCLUSION}

The responsiveness to training in each of the various age and sex groups was very varied. Young females were the most responsive to training in plantarflexion. Young males started from a higher baseline but did not gain strength any more rapidly than those in older age groups. Middle-aged females and elderly males did not respond at all in dorsiflexion. Increases in lower leg muscle strength following exercise training over 8 weeks were not reflected in MRI measures of muscle size or $T_{2}$ values.

\author{
Author affiliations \\ ${ }^{1}$ Arthritis and Musculoskeletal Medicine, University of Aberdeen, Aberdeen, \\ Scotland \\ ${ }^{2}$ Molecular Neuroscience, Institute of Neurology, University College London, \\ London, England \\ ${ }^{3}$ Aberdeen Biomedical Imaging Centre, University of Aberdeen, Aberdeen, \\ Scotland \\ ${ }^{4}$ Faculty of Medicine, Imperial College, London, England \\ ${ }^{5}$ Molecular Exercise Physiology Research Programme, University of Aberdeen, \\ Aberdeen, Scotland \\ ${ }^{6}$ TUSPFSP Fakultät für Sport und Gesundheitswissenschaften, München, \\ Germany \\ ${ }^{7}$ Department of Radiology, University of Cambridge School of Clinical \\ Medicine, Cambridge, England \\ ${ }^{8}$ Biomedical Physics Group, University of Exeter, Exeter, England
}

Acknowledgements We thank the MRI radiographers for performing the scanning, Dr Scott Semple for invaluable help in some of the pilot studies and Mr EC Stevenson for constructing the footrest used in the scanner. We are very grateful to the dedicated volunteers themselves who gave considerable amounts of time and showed remarkable enthusiasm to come in for scanning exercise and assessment during the course of this study.

Contributors RMA and JRM designed the study. JRM performed the initial analysis. RMA performed additional analysis and prepared the first draft of the paper. FJG was the senior radiologist, TWR was the MR physicist and together they directed the MRI and analysis. AR advised on the exercise training regime and $\mathrm{HW}$ on muscle function and physiology. MP and FG recruited the volunteers, put them through the exercise training, assessed their strength and ensured their adherence to the scanning protocols. ZW performed the image analysis to segment the images and make the MR measurements. All authors revised the paper critically for intellectual content and approved the final version. All authors agree to be accountable for the work and to ensure that any questions relating to the accuracy and integrity of the paper are investigated and properly resolved.

Funding This study was supported by an award (Ref: WHMSB_AU118) from the Translational Medicine Research Collaboration-a consortium made up of the Universities of Aberdeen, Dundee, Edinburgh and Glasgow, the four associated NHS Health Boards (Grampian, Tayside, Lothian and Greater Glasgow \& Clyde), Scottish Enterprise and Wyeth. The funder played no part in the design, execution, analysis or publication of this study.

\section{Competing interests None declared.}

Patient consent All the participants were healthy volunteers. No personal or identifying data on any of the participants are included in this paper

Ethics approval North of Scotland Research Ethics Committee.

Provenance and peer review Not commissioned; internally peer reviewed.
Open Access This is an Open Access article distributed in accordance with the Creative Commons Attribution Non Commercial (CC BY-NC 4.0) license, which permits others to distribute, remix, adapt, build upon this work noncommercially, and license their derivative works on different terms, provided the original work is properly cited and the use is non-commercial. See: http:// creativecommons.org/licenses/by-nc/4.0/

(C) Article author(s) (or their employer(s) unless otherwise stated in the text of the article) 2017. All rights reserved. No commercial use is permitted unless otherwise expressly granted.

\section{REFERENCES}

1. Roubenoff R, Hughes VA. Sarcopenia: current concepts. J Gerontol A Biol Sci Med Sci 2000;55:M716-24.

2. Bodine SC, Latres E, Baumhueter S, et al. Identification of ubiquitin ligases required for skeletal muscle atrophy. Science 2001;294:1704-8.

3. Psatha M, Wu Z, Gammie FM, et al. A longitudinal MRI study of muscle atrophy during lower leg immobilization following ankle fracture. J Magn Reson Imaging 2012;35:686-95.

4. Psatha M, Wu Z, Gammie FM, et al. A longitudinal study of muscle rehabilitation in the lower leg after cast removal using magnetic resonance imaging and strength assessment. International Biomechanics 2015;2:101-12.

5. Hatakenaka $\mathrm{M}$, Ueda $\mathrm{M}$, Ishigami $\mathrm{K}$, et al. Effects of aging on muscle T2 relaxation time: difference between fast- and slow-twitch muscles. Invest Radiol 2001;36:692-8.

6. Li K, Dortch RD, Welch EB, et al. Multi-parametric MRI characterization of healthy human thigh muscles at 3.0 T relaxation, magnetization transfer, fat/water, and diffusion tensor imaging. NMR Biomed 2014;27:1070-84.

7. Manini TM, Clark BC, Nalls MA, et al. Reduced physical activity increases intermuscular adipose tissue in healthy young adults. Am J Clin Nutr 2007;85:377-84

8. Marcus RL, Addison O, Kidde JP, et al. Skeletal muscle fat infiltration: impact of age, inactivity, and exercise. J Nutr Health Aging 2010;14:362-6.

9. Ababneh ZQ, Ababneh R, Maier SE, et al. On the correlation between $T(2)$ and tissue diffusion coefficients in exercised muscle: quantitative measurements at $3 \mathrm{~T}$ within the tibialis anterior. MAGMA 2008;21:273-8

10. Segal RL, Song AW. Nonuniform activity of human calf muscles during an exercise task. Arch Phys Med Rehabil 2005;86:2013-7.

11. Ploutz-Snyder LL, Tesch PA, Crittenden DJ, et al. Effect of unweighting on skeletal muscle use during exercise. J Appl Physiol 1995;79:168-75.

12. Borg GA. Psychophysical bases of perceived exertion. Med Sci Sports Exerc 1982;14:377-81.

13. Fukunaga T, Roy RR, Shellock FG, et al. Specific tension of human plantar flexors and dorsiflexors. J Appl Physiol 1996;80:158-65.

14. Johnson MA, Polgar J, Weightman D, et al. Data on the distribution of fibre types in thirty-six human muscles. an autopsy study. $J$ Neurol Sci 1973;18:111-29.

15. Greenhaff PL. The molecular physiology of human limb immobilization and rehabilitation. Exerc Sport Sci Rev 2006;34:159-63.

16. Reid RW, Foley JM, Jayaraman RC, et al. Effect of aerobic capacity on the T(2) increase in exercised skeletal muscle. J Appl Physiol 2001;90:897-902.

17. Goodpaster BH, Park SW, Harris TB, et al. The loss of skeletal muscle strength, mass, and quality in older adults: the health, aging and body composition study. J Gerontol A Biol Sci Med Sci 2006;61:1059-64.

18. Jones SW, Hill RJ, Krasney PA, et al. Disuse atrophy and exercise rehabilitation in humans profoundly affects the expression of genes associated with the regulation of skeletal muscle mass. Faseb $J$ 2004:18:1025-7.

19. Morse $\mathrm{Cl}$, Thom JM, Mian OS, et al. Muscle strength, volume and activation following 12-month resistance training in 70-year-old males. Eur J App/ Physiol 2005;95(2-3):197-204.

20. Borde R, Hortobágyi T, Granacher U. Dose-Response Relationships of Resistance Training in healthy old adults: a systematic review and Meta-Analysis. Sports Med 2015;45:1693-720.

21. Melnyk JA, Rogers MA, Hurley BF. Effects of strength training and detraining on regional muscle in young and older men and women. Eur J Appl Physiol 2009;105:929-38. 